\title{
Motor Cortical Network Excitability in Parkinson's Disease
}

\author{
Giorgio Leodori, MD, PhD, ${ }^{1,2}$ Maria Ilenia De Bartolo, MD, ${ }^{1}$ Andrea Guerra, MD, PhD, ${ }^{1}$ Andrea Fabbrini, MD, ${ }^{2}$ \\ Lorenzo Rocchi, MD, PhD, ${ }^{3,4}$ Anna Latorre, MD, PhD, ${ }^{3}$ (D) Giulia Paparella, MD, PhD, ${ }^{1}$ Daniele Belvisi, MD, PhD, ${ }^{1,2}$ \\ Antonella Conte, MD, PhD,,$^{1,2}$ Kailash P. Bhatia, FRCP, MD, ${ }^{3}$ (D) John C. Rothwell, PhD, ${ }^{3}$ (D) and \\ Alfredo Berardelli, MD ${ }^{1,2^{*}}$ (iD) \\ ${ }^{1}$ IRCCS Neuromed, Pozzilli, Italy \\ ${ }^{2}$ Department of Human Neurosciences, Sapienza University of Rome, Rome, Italy \\ ${ }^{3}$ Department of Clinical and Movement Neurosciences, UCL Queen Square Institute of Neurology, University College London, \\ London, United Kingdom \\ ${ }^{4}$ Department of Medical Sciences and Public Health, University of Cagliari, Cagliari, Italy
}

\begin{abstract}
Background: Motor impairment in Parkinson's disease (PD) reflects changes in the basal ganglia-thalamocortical circuit converging on the primary motor cortex (M1) and supplementary motor area (SMA). Previous studies assessed M1 excitability in PD using transcranial magnetic stimulation (TMS)-evoked electromyographic activity. TMS-evoked electroencephalographic activity may unveil broader motor cortical network changes in PD.

Objective: The aim was to assess motor cortical network excitability in PD.

Methods: We compared TMS-evoked cortical potentials (TEPs) from M1 and the pre-SMA between 20 PD patients tested off and on medication and 19 healthy controls ( $\mathrm{HCs})$ and investigated possible correlations with bradykinesia.

Results: Off PD patients compared to HCs had smaller P30 responses from the M1s contralateral $(\mathrm{M} 1+)$ and
\end{abstract}

ipsilateral (M1-) to the most bradykinetic side and increased pre-SMA N40. Dopaminergic therapy normalized the amplitude of $\mathrm{M} 1+$ and $\mathrm{M} 1-\mathrm{P} 30$ as well as preSMA N40. We found a positive correlation between M1+ P30 amplitude and bradykinesia in off PD patients.

Conclusions: Changes in M1 P30 and pre-SMA N40 in PD suggest that M1 excitability is reduced on both sides, whereas pre-SMA excitability is increased. The effect of dopaminergic therapy and the clinical correlation suggest that these cortical changes may reflect abnormal basal ganglia-thalamocortical activity. TMS electroencephalography provides novel insight into motor cortical network changes related to the pathophysiology of PD. (C) 2022 International Parkinson and Movement Disorder Society

Key Words: Parkinson's disease; motor cortex; supplementary motor area; bradykinesia; transcranial magnetic stimulation-electroencephalography
In Parkinson's disease (PD), nigrostriatal degeneration results in pathological activity of the basal gangliathalamocortical circuit, leading to abnormal control of the primary motor cortex (M1) and supplementary motor area (SMA). ${ }^{1-3}$ Animal models have suggested that the parkinsonian state is associated with a reduced

\footnotetext{
*Correspondence to: Prof. Alfredo Berardelli, Department of Human Neuroscience, Sapienza University of Rome, Viale dell'Università 30, 00185 Rome, Italy; E-mail: alfredo.berardelli@uniroma1.it
}

Relevant conflicts of interest/financial disclosures: Nothing to report. Financial agency: None.

Received: 1 June 2021; Revised: 13 December 2021; Accepted: 15 December 2021

Published online in Wiley Online Library

(wileyonlinelibrary.com). DOI: 10.1002/mds.28914 neuronal firing rate in $\mathrm{M}^{4,5}$ and the SMA, particularly in its rostral part (pre-SMA). ${ }^{6}$ Transcranial magnetic stimulation (TMS) studies based on the measurement of motor evoked potentials (MEPs) have suggested an association between PD and increased intracortical facilitation, ${ }^{7-9}$ decreased intracortical inhibition of M1 at rest, ${ }^{10-12}$ and reduced M1 excitability during contraction. ${ }^{13,14}$ However, TMS-evoked MEPs reflect the activation of specific circuits directly connected to pyramidal tract neurons ${ }^{15}$ rather than wider networks of neurons also activated by the TMS. Furthermore, MEPs cannot be used to assess the excitability of other cortical areas, such as the pre-SMA.

Cortical excitability can also be investigated by recording electroencephalography (EEG) potentials elicited by TMS (transcranial-evoked potentials [TEPs]). ${ }^{16-18}$ Compared to 
MEPs, TEPs assess the activation of a larger range of neurons in the stimulated cortex and reflect both local cortical excitability ${ }^{19-21}$ and cortical and subcortical connectivity. ${ }^{2-25}$ When TMS is delivered over M1, TEPs within $60 \mathrm{~ms}$ after stimulation have been consistently linked to local cortical excitability ${ }^{21,26,27}$ and have been termed P30, N45, and P60 according to their polarity and latency in millissecond. ${ }^{16,28}$ Although P30, N45, and $\mathrm{P} 60$ components are likely generated in the sensorimotor area, ${ }^{16,17,29-31}$ propagation of TMS-evoked activity along the cortico-subcortical loops backprojecting to the cortex also contributes to these components. ${ }^{20,24,32-35}$ Although there are no reports of TEPs from pre-SMA stimulation in healthy subjects, past studies have confirmed the validity of early TEPs to investigate excitability in other premotor areas. ${ }^{36,37}$ Therefore, TEPs may reveal changes in motor cortical activation in PD that cannot be identified when using MEPs as the outcome measure. $^{38}$

In the present study, we investigated whether TEPs could be used to identify changes in motor cortical network excitability in PD. To do so, we compared TEPs from M1 and the pre-SMA between PD patients and healthy controls (HCs). To test the effect of dopaminergic treatment on M1 and pre-SMA excitability and to determine whether cortical excitability changes are related to motor impairment, we investigated $\mathrm{PD}$ patients off $(\mathrm{OFF})$ and on $(\mathrm{ON})$ therapy and examined possible correlations between TEPs and clinical scores of motor impairment.

\section{Patients and Methods}

We consecutively enrolled 20 PD patients (12 men, aged $63.5 \pm 10.8$ years) and 19 age- and gendermatched HCs (10 men, aged $62.5 \pm 9.2$ years) from the outpatient unit at the Department of Human Neurosciences, Sapienza University of Rome, Italy (Table 1). HCs were enrolled from among nonconsanguineous relatives of patients. The study protocol was approved by the institutional review board and conducted in accordance with the latest revision of the Declaration of Helsinki. All patients provided written informed consent before participating in the study.

Clinical assessment included calculation of the levodopa equivalent daily dose, ${ }^{39}$ the Hoehn and Yahr $(\mathrm{H} \& \mathrm{Y})$ scale $^{40}$ the Movement Disorder Societysponsored Unified Parkinson's Disease Rating Scale (MDS-UPDRS), Part III, ${ }^{41}$ and the Montreal Cognitive Assessment (MoCA). ${ }^{42}$ For eligibility, all participants had to be right-handed as assessed by the Edinburgh Handedness Inventory. ${ }^{43}$ Inclusion criteria for patients also included PD diagnosis confirmed by a movement disorder expert neurologist based on international criteria $^{44,45}$ and the presence of bradykinesia with a minimum score of 2 on MDS-UPDRS items 3.4 to 3.6 related to the most affected upper limb, defined as the one showing the highest summed scores. Exclusion criteria included diagnosis of a neurological or psychiatric condition other than PD, advanced PD defined as $\mathrm{H} \& \mathrm{Y}$ stage IV or $\mathrm{V}$, and cognitive decline (MoCA score $<26$ ) due to possible difficulties in compliance and medication withdrawal.

A Magstim SuperRapid stimulator (Magstim Company, Whitland, United Kingdom) connected to a 70$\mathrm{mm}$ figure-of-eight coil was used to deliver TMS in the real stimulation condition. Sham stimulation was delivered by a 70-mm figure-of-eight sham coil (Magstim Company), which produces an auditory percept similar to real TMS without cortical activation. Neuronavigation (SofTaxic, EMS, Bologna, Italy) with an optical tracking system (Polaris Vicra, Northern Digital Inc., Waterloo, Canada) was used to monitor coil positioning for all stimulation sites. Using the neuronavigation system, we sampled 23 points from the scalp of each participant and adapted the reconstructed brain to Montreal Neurological Institute (MNI) space using nonlinear fitting. In all participants, the M1s of both sides were stimulated over the hotspot evoking the largest MEP in the contralateral first dorsal interosseous (FDI) muscle with posterior-anterior current direction. Resting motor threshold (RMT) was defined separately for each M1 as the minimum intensity required to elicit MEPs of $\geq 50 \mu \mathrm{V}$ peak-to-peak amplitude in at least 5 of 10 consecutive trials. For pre-SMA stimulation, the coil was placed with the handle pointing backward, parallel to the interhemispheric fissure and centered over the MNI coordinates $x=0, \mathrm{y}=10, z=68$. Coordinates for pre-SMA targeting were provided by previous studies ${ }^{46,47}$ but shifted to the midline (ie, $x=0$ ) to produce focal activation of medial Brodmann area 6 (Figure S1A).

Electromyography (EMG) activity from the FDI was recorded through pairs of $\mathrm{Ag} / \mathrm{AgCl}$ surface electrodes arranged in a belly-tendon montage. EMG signal was bandpass filtered $(10-1000 \mathrm{~Hz})$, amplified $(\times 1000)$ (D360, Digitimer, Welwyn Garden City, United Kingdom), digitized at $5 \mathrm{kHz}$ (CED1401, Cambridge Electronic Design, Cambridge, United Kingdom), and stored on a computer. EEG was recorded using a TMScompatible amplifier (NeurOne, Bittium, Oulu, Finland) from 32 passive electrodes mounted on an elastic cap (BrainCap, EASYCAP, Wörthsee, Germany) according to the international 10-20 system: Fp1-Fp2AFz-F7-F3-Fz-F4-F8-FC5-FC1-FCz-FC2-FC6-T7-C3Cz-C4-T8-TP9-CP5-CP1-CP2-CP6-TP10-P7-P3-Pz-P4P8-O1-O2-Iz. EEG signal was bandpass filtered (DC$2.5 \mathrm{kHz}$ ) and digitized at $10 \mathrm{kHz}$. All electrodes were grounded to $\mathrm{Fpz}$ and online referenced to POz. Impedance for each channel was kept below $5 \mathrm{k} \Omega$. Participants wore ear defenders (signal-to-noise ratio $=30)^{48,49}$ on top of earphones continuously playing a noise designed to mask 
TABLE 1 Patient demographics and clinical information



Abbreviations: Hoehn \& Yahr; LEDD, levodopa equivalent daily dose; MDS-UPDRS, Movement Disorder Society-sponsored Unified Parkinson's Disease Rating Scale; R, right; L, left; MoCA, Montreal Cognitive Assessment score; SD, standard deviation.

$\star$ Sum of scores $3.4,3.5$, and 3.6 of the MDS-UPDRS for the upper limb showing the highest score.

the TMS click. ${ }^{50}$ In addition, a 0.5 -cm foam layer was placed under the coil to minimize bone conduction of the TMS click and scalp sensation caused by coil vibration. $^{48,51}$

Participants were comfortably seated on a chair designed for TMS (EMS), with their forearms resting on armrests. They were instructed to stay relaxed and look at a black cross displayed on a PC screen at a distance of $70 \mathrm{~cm}$. FDI EMG activity was continuously monitored online, and participants were instructed to keep their hands relaxed. TMS trials showing EMG activity above $0.05 \mathrm{mV}$ in the first second before each pulse were excluded online (always less than $5 \%$ of trials). Single TMS pulses were delivered during EEG and EMG recording using an intensity equal to $110 \%$ RMT during three blocks of real and three blocks of sham TMS over M1 left, M1 right, and the pre-SMA. Sham stimulation was performed on 15 patients and 13 HCs. Intensities were defined by asking each participant to report the maximal stimulation output value that best matched the perception of loudness of the TMS click produced by real stimulation on the same site and were on average $5 \%-10 \%$ higher than real stimulation values. However, participants were not informed about the presence of sham stimulation. The order of the blocks was randomized for each participant. One hundred TMS pulses were delivered in each block, with an interpulse interval of $1.65 \mathrm{~s} \pm 10 \%$, a stimulation frequency that does not affect longitudinally recorded TEPs. ${ }^{22}$ We tested PD patients ON and OFF in two separate sessions performed 2-4 weeks apart and in a counterbalanced order across patients. 
LEO DOR I E T A L

For the OFF session, patients discontinued their antiparkinsonian medications at least 24 hours before the examination. HCs participated in a single experimental session.

We measured mean background muscular activity and peak-to-peak MEP amplitude for each participant (see Supplementary Material). TMS-EEG signal preprocessing was performed using the EEGLAB ${ }^{52}$ and TMS-EEG Signal Analyser (TESA) $)^{53,54}$ open-source Matlab (v.2017b) toolboxes (see Supplementary Material). Cleaned TMS-EEG epochs from -1.2 second before to 1.2 second after TMS pulses were converted into reference-free current source density (CSD) estimates using the "CSD" 55 open-source Fieldtrip" ${ }^{56}$ toolbox. Final TEPs were obtained by averaging TMS-EEG epochs (average number $93 \pm 3$ ) in each block. In patients, we defined the most affected M1 $(\mathrm{M} 1+)$ and the least affected M1 (M1-), respectively, as the one contralateral and ipsilateral to the most affected upper limb. To obtain a homogeneous representation of scalp potential across groups, EEG electrode positions were mirrored across the two hemispheres in patients whose $\mathrm{M} 1+$ was the right one. The same was performed to compare TEPs from M1 left and M1 right in HCs. We compared M1 left and M1 right of HCs, respectively, with $\mathrm{M} 1+$ and M1- of PD patients.
Statistical analysis was performed using SPSS (version 25.0.0, IBM, Armonk, NY, US). Between-group (PD, $\mathrm{HC})$ differences regarding age and gender were investigated using Mann-Whitney $U$ and $\chi^{2}$ tests. Paired or unpaired $t$ tests were used to investigate differences in RMT, baseline EMG, and MEP amplitude between HCs and OFF and ON patients and to clarify changes in MDS-UPDRS scores according to medication (ON vs. OFF) conditions.

For each stimulation site, we computed grand average TEPs from all electrodes by averaging the mean TEPs in HCs and OFF and ON patients. We also computed local TEPs by averaging signals from the three channels closest to the stimulation site in each group (M1 left/ $\mathrm{M} 1+$ : C3-Cz-FC1; M1 right/M1-: C4-Cz-FC2; preSMA: FCz-Fz-Cz). For each stimulation site, TEP components were defined as positive (P, or sources according to CSD estimates) or negative ( $\mathrm{N}$, or sinks according to CSD estimates) when showing a reversed U shape or a U shape on local TEPs, respectively. Analysis was limited within $60 \mathrm{~ms}$ after stimulation because this latency range has been more consistently related to local cortical excitability ${ }^{21}$ and may also reflect corticosubcortical dynamics. ${ }^{20,32-34,57,58}$

We measured the peak latency of each TEP component as the time point of the maximal baseline-to-peak
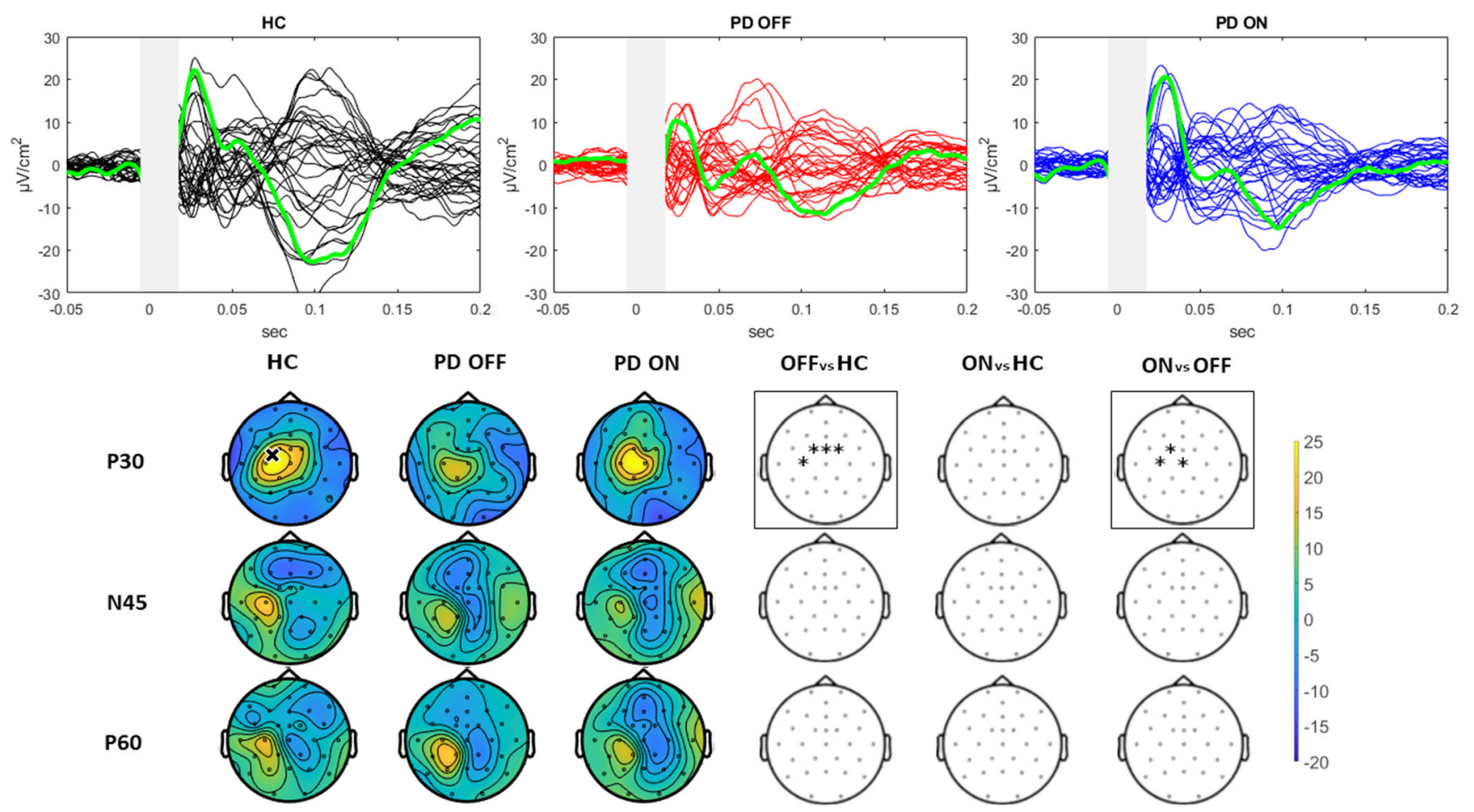

OFFus $\mathrm{HC}$
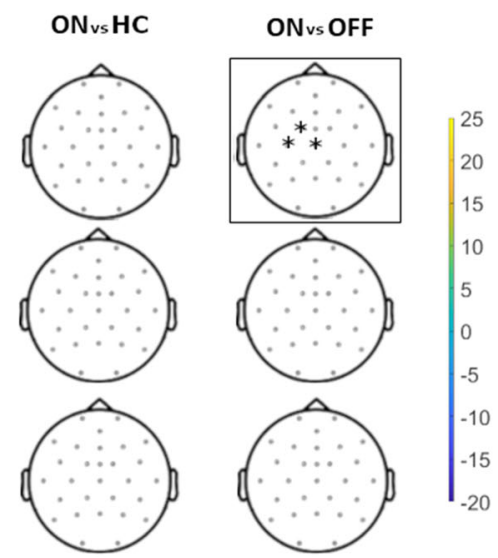

FIG. 1. TEPs from stimulation of the M1 contralateral to the most bradykinetic upper limb. Above: grand average butterfly plots of CSD-transformed TEPs from all channels; green lines represent the signal close to the stimulation site (average across C3, FC1, Cz). Below: topo plots of the investigated times of interest; black cross shows approximate stimulation site; yellow represents positive CSD values (sources); blue represents negative values (sinks); squares highlight significant comparisons; asterisks highlight channels significantly different in cluster-based permutation analysis. CSD, current source density; TEP, TMS-evoked cortical potential. [Color figure can be viewed at wileyonlinelibrary.com] 

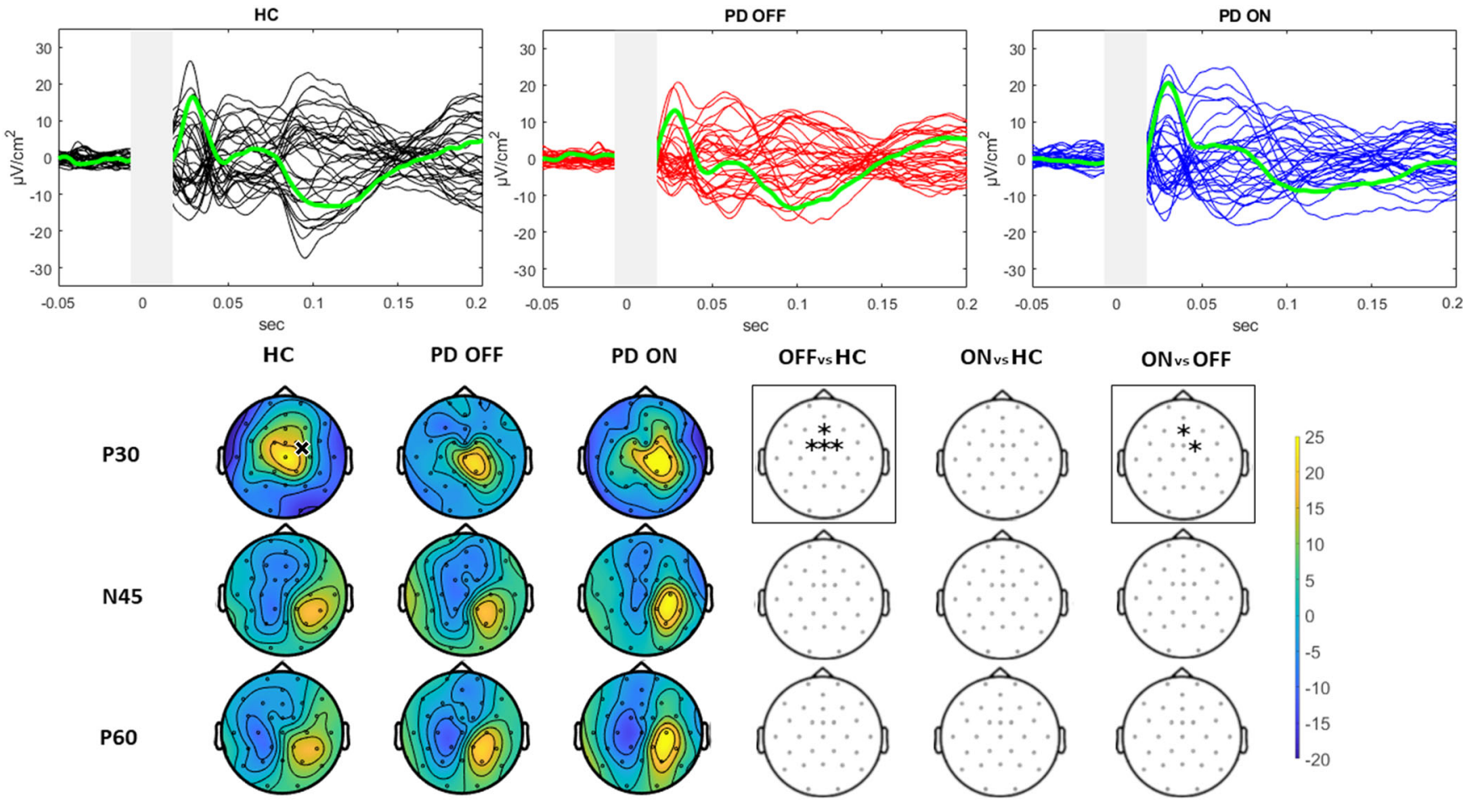

FIG. 2. TEPs from stimulation of the M1 ipsilateral to the most bradykinetic upper limb. Above: grand average butterfly plots of CSD-transformed TEPs from all channels; green lines represent the signal close to the stimulation site (average across C4, FC2, Cz). Below: topo plots of the investigated times of interest; black cross shows approximate stimulation site; yellow represents positive CSD values (sources); blue represents negative values (sinks); squares highlight significant comparisons; asterisks highlight channels significantly different in cluster-based permutation analysis. CSD, current source density; TEP, TMS-evoked cortical potential. [Color figure can be viewed at wileyonlinelibrary.com]
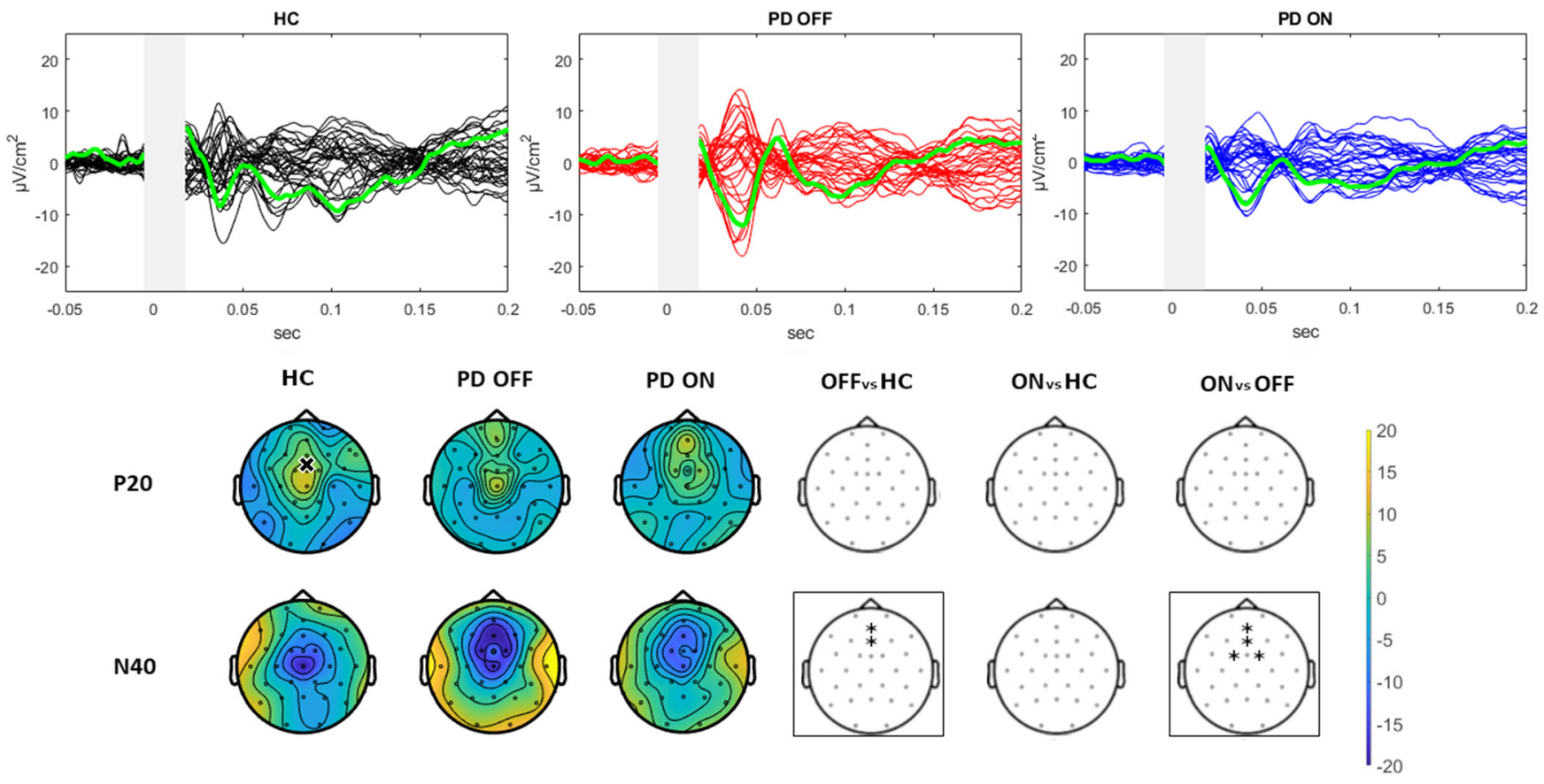

FIG. 3. TEPs from pre-SMA stimulation. Above: grand average butterfly plots of CSD-transformed TEPs from all channels; green lines represent the signal close to the stimulation site (average across $\mathrm{Cz}, \mathrm{FCz}, \mathrm{Fz}$ ). Below: topo plots of the investigated times of interest; black cross shows approximate stimulation site; yellow represents positive CSD values (sources); blue represents negative values (sinks); squares highlight significant comparisons; asterisks highlight channels significantly different in cluster-based permutation analysis. CSD, current source density; SMA, supplementary motor area; TEP, TMS-evoked cortical potential. [Color figure can be viewed at wileyonlinelibrary.com] 
LEODOR I E T A L

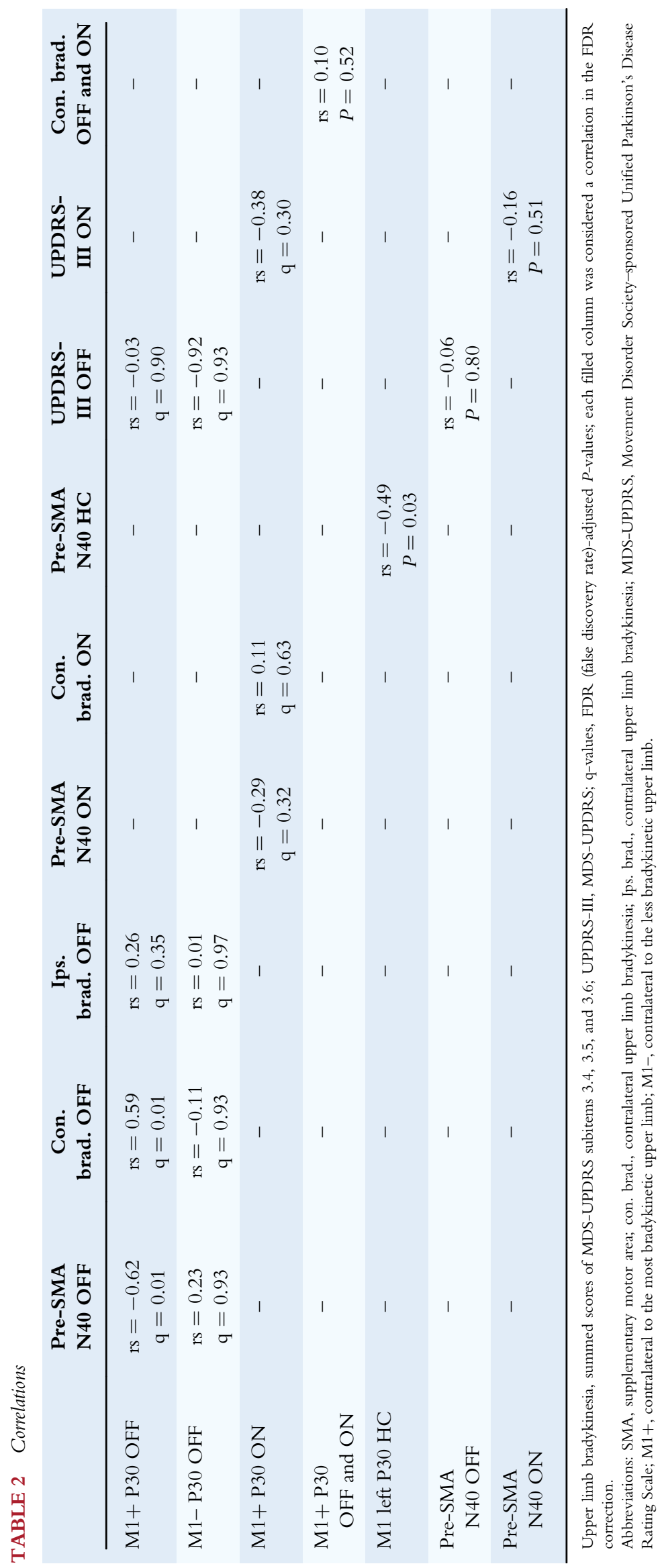


amplitude value on grand average TEPs across groups, and we defined the following time window of interest (TOI) for M1-P30 (28 $\pm 5 \mathrm{~ms}), \mathrm{N} 45$ (46 $\pm 5 \mathrm{~ms})$, and P60 (62 $\pm 5 \mathrm{~ms})$-and for the pre-SMA: P20 $(19 \pm 5 \mathrm{~ms})$ and N40 (39 $\pm 5 \mathrm{~ms})$ (Figure S1B). TEP components in each TOI were compared between groups (HCs vs. $\mathrm{PD}$ ) and conditions (OFF vs. ON, and left vs. right in HCs) separately for real and sham TMS using cluster-based permutation testing as implemented in Fieldtrip (clusters were significant with a $P$-value $<0.05$ false discovery rate [FDR] corrected for multiple

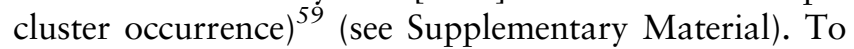
test whether the interpulse interval we used influenced TEPs, we measured whether TEP amplitude changed during M1+ block (see Supplementary Material). We assessed Spearman's correlations on average TEP amplitude across the channels in the clusters significantly affected by parkinsonian state between different stimulation sites to test possible common mechanisms underlying TEP abnormalities in PD. We assessed the same correlations in HCs to test whether the results were specific for PD. We also computed correlations between these TEPs and bradykinesia scores from both upper limbs to test the side specificity of our results. We corrected for multiple correlations by computing FDR with the one-stage method ${ }^{60}$ (see Supplementary Material). All values are expressed as mean \pm standard deviation.

\section{Results}

Mean age of PD patients and HCs did not differ statistically $(\mathrm{U}=176.5, P=0.71)$. There was no significant difference in the proportion of men/women between patients and HCs $\left(\chi^{2}(1)=0.22, P=0.64\right)$. Patients showed significantly lower MDS-UPDRS scores in the ON versus OFF condition $(\mathrm{t}(19)=9.94$, $P<0.001$ ) (Table 1). RMT, background EMG activity, and MEP amplitude were similar between groups and conditions (see Table S1).

Average TEPs showed that the first components (P30 for M1 stimulation and P20 for pre-SMA stimulation) had maximal activity close to the stimulation site (Figs. 1-3). After M1 stimulation of both sides, P30 topography showed a centro-frontal source predominant over the stimulated hemisphere. In N45 and P60 TOIs, the dominant source of activity progressively moved posteriorly over centro-parietal sites, and contralateral frontal and parietal sinks were noted (Figs. 1 and 2).

After pre-SMA stimulation, there was a medial cfrontal source in the P20 TOI and a medial central sink in the N40 TOI (Fig. 3).

Considering the M1 of both sides, there was significantly smaller activity in the P30 centro-frontal source in OFF patients than in HCs (M1+ cluster: C3-FCzFC1-FC2; M1- cluster: FCz-Fz-FC1-FC2) (Fig. 1). We found no differences in TEPs evoked by left and right M1 stimulation in HCs. After pre-SMA stimulation, OFF PD patients showed significantly larger activity in the N40 sink over centro-frontal areas $(\mathrm{Fz}-\mathrm{AFz})$ than HCs (Fig. 3). N45 and P60 from each M1 and preSMA P20 were comparable between groups.

We found significant negative correlations between M1+ P30 amplitude and pre-SMA N40 amplitude in OFF PD patients and HCs, whereas no significant correlation was found in $\mathrm{ON}$ patients (Figure S2) (Table 2).

Sham stimulation of M1 on both sides and the preSMA induced no detectable TEPs, and sham blocks were similar between groups and medication conditions (Figures S3-S5).

After M1 stimulation on both sides, patients had significantly higher amplitude in the P30 centro-frontal source (M1+: C3-Cz-FC1; M1-: Fz-FC2) in the ON versus OFF condition. We found no differences in M1 $\mathrm{P} 30, \mathrm{~N} 45$, or P60 on either side between $\mathrm{ON}$ patients and HCs. Dopaminergic treatment did not affect M1 N45 or P60 of either side (Figs. 1 and 2). The pre-SMA $\mathrm{N} 40$ sink over the medial frontal areas $(\mathrm{Fz}, \mathrm{AFz}, \mathrm{FC}$, and FC2) was significantly smaller in the $\mathrm{ON}$ versus OFF condition, and we found no differences in preSMA N40 between patients ON and HCs. Dopaminergic treatment did not affect pre-SMA P20 amplitude (Fig. 3). All significant clusters survived FDR correction (Table S2). Cluster-based analysis in both HCs and OFF patients showed no significant differences in TEPs between the first, the second, and the last third of TMS epochs.

In OFF patients, M1+ P30 amplitude showed a strong positive correlation with bradykinesia scores in the contralateral more affected upper limb but no correlation with the ipsilateral/less-affected upper limb. No significant correlations were found in the ON condition, when whole OFF and ON values were considered together, or for M1- P30. Finally, no significant correlations were found between MDS-UPDRS-III scores and M1+ and M1- P30 amplitude or pre-SMA N40 amplitude (Figure S2) (Table 2).

\section{Discussion}

The present study revealed several TEP changes in PD. In OFF PD patients, the P30 component of TEPs evoked by M1 stimulation of both hemispheres was smaller than in HCs. In contrast, N40 TEPs from preSMA stimulation were larger in OFF patients compared to HCs. In OFF patients, there was a significant correlation between the amplitudes of M1 P30 and pre-SMA N40. Finally, sham stimulation did not elicit TEPs from 
LEODORI ET A L

either site. Dopaminergic treatment increased the size of P30 evoked by stimulation of M1 in both hemispheres, whereas the size of pre-SMA N40 decreased. M1 P30 and pre-SMA N40 sizes did not differ between ON PD patients and HCs. Finally, in OFF PD patients there was a significant correlation between the size of $\mathrm{M} 1+$ P30 and contralateral bradykinesia clinical scores, whereas no correlations were found between M1 and pre-SMA TEPs and MDS-UPDRS-III score.

We excluded several confounding factors. Previous work had shown that interpulse intervals around 1 second can produce long-term changes in MEPs. ${ }^{61}$ However, we found that TEP amplitude obtained by averaging the first third of TMS epochs did not significantly differ from TEP amplitude observed in the second and last third of trials for HCs or OFF patients. This analysis suggests that possible short-term effects did not confound our results. Our results were likely not confounded by EEG responses caused by auditory input because sham stimulation evoked no significant activity. ${ }^{48,49,51}$ This result also shows that the concurrent use of a layer of foam under the coil, ear defenders, and noise masking effectively suppresses auditory responses even for suprathreshold TMS intensities. ${ }^{48,49}$ The possibility that our TEPs reflect nonspecific sensory responses is also reduced by the observation that TEPs showed topographic specificity. ${ }^{18,21,22,48}$ Finally, although TEPs evoked by stimulation intensities above motor threshold may be contaminated by sensory reafferents associated with muscle contraction, our results mainly concern latencies preceding this possible confounding factor, ${ }^{62,63}$ and because MEP amplitudes were similar between groups and conditions, sensory reafferents could not explain the TEP differences we found.

Alterations in TEP size from the M1 and pre-SMA suggest that PD is associated with abnormalities in cortical motor network excitability. TEPs are generated by a summation of excitatory and inhibitory postsynaptic potentials generated by the activity of cortical pyramidal neurons and interneurons and reflect cortical excitability. ${ }^{16-22}$ Animal intracortical recordings from layer 5 showed a significant increase in the firing rate of pyramidal neurons in the P30 time range ${ }^{33}$ and reduced pyramidal neuron activity in animal models of PD. ${ }^{4,5,64}$ Therefore, the decreased P30 amplitude after stimulation of M1 on both sides likely reflects reduced excitability of M1 pyramidal neurons in OFF PD patients. ${ }^{3-5}$ Besides local cortical excitability, TEPs reflect the connectivity of the stimulated area with other cortical and subcortical areas, and therefore, TEP abnormalities may reflect motor network changes in PD. ${ }^{22-25}$ Corticosubcortical loops involving thalamocortical projections may contribute to TEPs in the $10-$ to $50-\mathrm{ms}$ range, ${ }^{20,32-34}$ and the P30 distribution we observed is consistent with it being the cortical source of a radial dipole with a subcortical sink. We speculate that one circuit that contributes to P30 is an excitatory corticosubcortical network through the basal ganglia and thalamus and back to the cortex. ${ }^{57,58}$ Reduced excitability in this network in PD would result in reduced thalamocortical excitation and reduced P30., ${ }^{1,2}$ However, degeneration of thalamic dopamine innervation has recently been described in PD animal models, and therefore, P30 changes in PD may reflect thalamic changes unrelated to basal ganglia abnormalities. ${ }^{65}$ Reduced M1 P30 is consistent with the reduced motor-related M1 activation reported by neuroimaging studies ${ }^{66}$ and provides evidence of PD-related neural dysfunction that is not confounded by differences in motor task performance because TEPs were recorded at rest.

The observed reduction in M1 P30 amplitude in PD patients confirms the results of a recent non-shamcontrolled TMS-EEG study that was performed in a smaller group of advanced PD patients implanted with deep brain stimulation. ${ }^{67}$

Our finding of increased pre-SMA N40 amplitude suggests pre-SMA hyperexcitability in $\mathrm{PD}$, which is consistent with the hyperactivation reported by neuroimaging studies. ${ }^{66}$ N40 distribution was compatible with a radial dipole, with centro-frontal negativity generated by corticofugal outputs. Therefore, increased pre-SMA N40 amplitude may reflect changes along cortico-subcortical circuits in PD. ${ }^{66,68}$

Our finding of increased pre-SMA TEPs in PD contrasts with that recently reported in a similar study. ${ }^{46}$ However, while we centered the coil over the midline, Casarotto and colleagues stimulated a more lateral area, eliciting TEPs that may receive larger contributions from the dorsal premotor cortex (PMd).

We found that dopaminergic therapy normalized P30 elicited by M1 stimulation of both hemispheres as well as pre-SMA N40. Also, dopaminergic treatment modulated the correlation between M1 P30 and pre-SMA N40 observed in OFF PD patients. This result confirms that TEPs provide a useful indicator of dopaminergic therapy effect on motor cortical excitability in PD. Our finding that dopaminergic therapy normalized M1 P30 and pre-SMA N40 amplitudes suggests that abnormalities in these components in PD reflect motor cortical changes due to abnormal basal ganglia-thalamocortical activity. ${ }^{1,2}$

Correlation analyses demonstrated that OFF PD patients who were more bradykinetic had larger M1+ P30, whereas no correlations were found in ON PD patients, even when whole ON and OFF state values were considered together. The lack of correlation in the $\mathrm{ON}$ condition suggested that reduced $\mathrm{P} 30$ size in PD is not a direct marker of bradykinesia. Because P30 reduction inversely correlated with contralateral bradykinesia in OFF PD patients, decreased P30 may reflect M1 compensatory mechanisms to maintain motor output in 
the OFF state. ${ }^{69}$ Alternatively, the lack of correlation in the $\mathrm{ON}$ condition could be due to a smaller dispersion of bradykinesia scores in the ON versus OFF condition that may have limited the sensitivity of the correlation analysis in ON PD patients. However, this possibility is unlikely because Table 1 shows similar standard deviation of bradykinesia scores between OFF and ON conditions.

We acknowledge some limitations. Although we found that the short interpulse interval we used likely did not affect TEP amplitude, our MEP analysis could have been confounded by possible mechanisms related to the dependence of consecutive responses. ${ }^{70}$ Future studies using interpulse intervals $>5$ seconds are necessary for a better comparison between TMS-EEG and TMS-EMG responses. Although our sham stimulation produced a tactile vibratory sensation, somatosensory co-stimulation was not completely controlled for in sham blocks. However, somatosensory co-stimulation does not contribute significantly to the early TEPs considered in the present study. ${ }^{48,71}$ Because we did not use individual MRI neuronavigation, variability in preSMA and SMA-proper locations may have confounded TEP results due to distinctive functional changes occurring in the pre-SMA and SMA-proper in PD. ${ }^{64}$ However, because we centered our coil over pre-SMA coordinates that did not overlap with the SMAproper, ${ }^{46,72}$ and because our estimated electric field maximal values (Figure S1) were within the boundaries of the pre-SMA maximal probability area, ${ }^{72}$ we believe that our methods resulted in high spatial selectivity for pre-SMA activation with limited confounding due to SMA coactivation. Also, the increase in pre-SMA TEPs is in line with the pre-SMA hyperactivation previously reported, ${ }^{66}$ supporting the conclusion that our results likely reflect pre-SMA excitability. Although bilateral pre-SMA activation in our study could have limited our sensitivity to identify asymmetrical pre-SMA involvement in PD, a recent meta-analysis suggested bilateral pre-SMA hyperactivation in PD. ${ }^{66}$ In addition, TEPs elicited by more lateral coil placement would have been confounded by the mixed activation of different premotor areas. We acknowledge that since the reported effect sizes were calculated a posteriori, they are likely overestimated, and therefore, studies with larger samples and hypothesis-driven designs are warranted to confirm our results and reach more definitive conclusions. Studies on patients with other movement disorders are also necessary to test the specificity of our results in PD.

In conclusion, our TMS-EEG study demonstrated motor cortical network excitability changes in PD and their relationship with nigrostriatal degeneration, findings that have important pathophysiological and clinical implications. We demonstrated that TEPs can be used to identify motor cortical activation changes in PD without the need for the motor-related tasks used in other neuroimaging studies. In addition, our study showed that TEPs reveal changes in the excitability of neuronal populations that differ from those found using MEPs and that may partially reflect changes in circuits involving subcortical structures sensitive to dopaminergic status. Further studies are needed to clarify the relevance of our results to PD pathophysiology and their clinical correlates.

\section{Data Availability Statement}

The data that support the findings of this study are available from the corresponding author upon reasonable request.

\section{References}

1. DeLong MR. Primate models of movement disorders of basal ganglia origin. Trends Neurosci 1990;13:281-285.

2. Wichmann T. Changing views of the pathophysiology of parkinsonism. Mov Disord 2019;34:1130-1143.

3. Underwood CF, Parr-Brownlie LC. Primary motor cortex in Parkinson's disease: functional changes and opportunities for neurostimulation. Neurobiol Dis 2021;147:105159

4. Pasquereau B, Turner RS. Primary motor cortex of the parkinsonian monkey: differential effects on the spontaneous activity of pyramidal tract-type neurons. Cereb Cortex 2011;21:1362-1378.

5. Pasquereau B, DeLong MR, Turner RS. Primary motor cortex of the parkinsonian monkey: altered encoding of active movement. Brain 2016;139:127-143.

6. Escola L, Michelet T, Macia F, Guehl D, Bioulac B. Disruption of information processing in the supplementary motor area of the MPTP-treated monkey: a clue to the pathophysiology of akinesia? Brain 2003;126:95-114.

7. Ni Z, Bahl N, Gunraj CA, Mazzella F, Chen R. Increased motor cortical facilitation and decreased inhibition in Parkinson disease. Neurology 2013;80:1746-1753.

8. Guerra A, Suppa A, D'Onofrio V, et al. Abnormal cortical facilitation and L-dopa-induced dyskinesia in Parkinson's disease. Brain Stimul 2019;12:1517-1525.

9. Shirota Y, Ohminami S, Tsutsumi R, Terao Y, Ugawa Y, Tsuji S, Hanajima R. Increased facilitation of the primary motor cortex in de novo Parkinson's disease. Parkinsonism Relat Disord 2019;66: 125-129.

10. Ammann C, Dileone M, Pagge C, et al. Cortical disinhibition in Parkinson's disease. Brain 2020;143:3408-3421.

11. Belvisi D, Fabbrini A, De Bartolo MI, et al. The pathophysiological correlates of Parkinson's disease clinical subtypes. Mov Disord 2021;36:370-379.

12. Latorre A, Rocchi L, Berardelli A, Bhatia KP, Rothwell JC. The interindividual variability of transcranial magnetic stimulation effects: implications for diagnostic use in movement disorders. Mov Disord 2019;34:936-949.

13. Berardelli A, Rona S, Inghilleri M, Manfredi M. Cortical inhibition in Parkinson's disease. A study with paired magnetic stimulation. Brain 1996;119(pt 1):71-77.

14. Berardelli A, Rothwell JC, Thompson PD, Hallett M. Pathophysiology of bradykinesia in Parkinson's disease. Brain 2001; 124:2131-2146.

15. Di Lazzaro V, Oliviero A, Pilato F, et al. The physiological basis of transcranial motor cortex stimulation in conscious humans. Clin Neurophysiol 2004;115:255-266.

16. Ilmoniemi RJ, Virtanen J, Ruohonen J, et al. Neuronal responses to magnetic stimulation reveal cortical reactivity and connectivity. Neuroreport 1997;8:3537-3540. 
17. Lioumis P, Kičić D, Savolainen P, Mäkelä JP, Kähkönen S. Reproducibility of TMS-evoked EEG responses. Hum Brain Mapp 2009; 30:1387-1396.

18. Tremblay S, Rogasch NC, Premoli I, et al. Clinical utility and prospective of TMS-EEG. Clin Neurophysiol 2019;130:802-844.

19. Paus T, Sipila PK, Strafella AP. Synchronization of neuronal activity in the human primary motor cortex by transcranial magnetic stimulation: an EEG study. J Neurophysiol 2001;86:1983-1990.

20. Mäki H, Ilmoniemi RJ. EEG oscillations and magnetically evoked motor potentials reflect motor system excitability in overlapping neuronal populations. Clin Neurophysiol 2010;121:492-501.

21. Leodori G, Thirugnanasambandam N, Conn H, Popa T, Berardelli A, Hallett M. Intracortical inhibition and surround inhibition in the motor cortex: a TMS-EEG study. Front Neurosci 2019; 13:612.

22. Casarotto S, Romero Lauro LJ, Bellina V, et al. EEG responses to TMS are sensitive to changes in the perturbation parameters and repeatable over time. PLoS One 2010;5:e10281

23. Casula EP, Maiella M, Pellicciari MC, et al. Novel TMS-EEG indexes to investigate interhemispheric dynamics in humans. Clin Neurophysiol 2020;131:70-77.

24. Peters JC, Reithler J, de Graaf TA, Schuhmann T, Goebel R, Sack AT. Concurrent human TMS-EEG-fMRI enables monitoring of oscillatory brain state-dependent gating of cortico-subcortical network activity. Commun Biol 2020;3:40

25. Ozdemir RA, Tadayon E, Boucher P, et al. Individualized perturbation of the human connectome reveals reproducible biomarkers of network dynamics relevant to cognition. Proc Natl Acad Sci U S A 2020;117:8115-8125.

26. Ferreri F, Pasqualetti $P$, Määttä $S$, et al. Human brain connectivity during single and paired pulse transcranial magnetic stimulation. Neuroimage 2011;54:90-102.

27. Cash RFH, Noda Y, Zomorrodi R, et al. Characterization of glutamatergic and gabaa-mediated neurotransmission in motor and dorsolateral prefrontal cortex using paired-pulse TMS-EEG. Neuropsychopharmacology 2017;42:502-511.

28. Komssi S, Aronen HJ, Huttunen J, et al. Ipsi- and contralateral EEG reactions to transcranial magnetic stimulation. Clin Neurophysiol 2002;113:175-184.

29. Komssi S, Kähkönen S. The novelty value of the combined use of electroencephalography and transcranial magnetic stimulation for neuroscience research. Brain Res Rev 2006;52:183-192.

30. Bonato C, Miniussi C, Rossini PM. Transcranial magnetic stimulation and cortical evoked potentials: a TMS/EEG co-registration study. Clin Neurophysiol 2006;117:1699-1707.

31. Ahn S, Fröhlich F. Pinging the brain with transcranial magnetic stimulation reveals cortical reactivity in time and space. Brain Stimul 2021;14:304-315.

32. Van Der Werf YD, Sadikot AF, Strafella AP, Paus T. The neural response to transcranial magnetic stimulation of the human motor cortex. II. Thalamocortical contributions. Exp Brain Res 2006;175: 246-255.

33. Li B, Virtanen JP, Oeltermann A, et al. Lifting the veil on the dynamics of neuronal activities evoked by transcranial magnetic stimulation. Elife 2017;6.

34. Pellicciari MC, Bonnì S, Ponzo V, et al. Dynamic reorganization of TMS-evoked activity in subcortical stroke patients. Neuroimage 2018;175:365-378.

35. Rosanova M, Casali A, Bellina V, Resta F, Mariotti M, Massimini M. Natural frequencies of human corticothalamic circuits. J Neurosci 2009;29:7679-7685.

36. Ferrarelli F, Massimini M, Sarasso S, et al. Breakdown in cortical effective connectivity during midazolam-induced loss of consciousness. Proc Natl Acad Sci U S A 2010;107:2681-2686.

37. Ferrarelli F, Massimini M, Peterson MJ, et al. Reduced evoked gamma oscillations in the frontal cortex in schizophrenia patients: a TMS/EEG study. Am J Psychiatry 2008;165:996-1005.

38. Leodori G, Belvisi D, De Bartolo MI, et al. Re-emergent tremor in Parkinson's disease: the role of the motor cortex. Mov Disord 2020; 35:1002-1011.
39. Tomlinson CL, Stowe R, Patel S, Rick C, Gray R, Clarke CE. Systematic review of levodopa dose equivalency reporting in Parkinson's disease. Mov Disord 2010;25:2649-2653.

40. Hoehn MM, Yahr MD. Parkinsonism:onset, progression, and mortality. Neurology 1967;17:427-427.

41. Goetz CG, Tilley BC, Shaftman SR, et al. Movement Disorder Society-sponsored revision of the unified Parkinson's disease rating scale (MDS-UPDRS):scale presentation and clinimetric testing results. Mov Disord 2008;23:2129-2170.

42. Nasreddine ZS, Phillips NA, Bédirian V, et al. The Montreal cognitive assessment, MoCA: a brief screening tool for mild cognitive impairment. J Am Geriatr Soc 2005;53:695-699.

43. Oldfield RC. The assessment and analysis of handedness: the Edinburgh inventory. Neuropsychologia 1971;9:97-113.

44. Berardelli A, Wenning GK, Antonini A, et al. EFNS/MDS-ES/ENS [corrected] recommendations for the diagnosis of Parkinson's disease. Eur J Neurol 2013;20:16-34.

45. Postuma RB, Berg D, Stern M, et al. MDS clinical diagnostic criteria for Parkinson's disease. Mov Disord 2015;30:1591-1601.

46. Casarotto S, Turco F, Comanducci A, et al. Excitability of the supplementary motor area in Parkinson's disease depends on subcortical damage. Brain Stimul 2019;12:152-160.

47. Mayka MA, Corcos DM, Leurgans SE, Vaillancourt DE. Threedimensional locations and boundaries of motor and premotor cortices as defined by functional brain imaging. Neuroimage 2006;31: 1453-1474.

48. Rocchi L, Di Santo A, Brown K, et al. Disentangling EEG responses to TMS due to cortical and peripheral activations. Brain Stimul 2021;14:4-18.

49. Mancuso M, Sveva V, Cruciani A, et al. Transcranial evoked potentials can be reliably recorded with active electrodes. Brain Sci 2021; 11:1-16.

50. Massimini M, Ferrarelli F, Huber R, Esser SK, Singh H, Tononi G. Breakdown of cortical effective connectivity during sleep. Science 2005;309:2228-2232.

51. ter Braack EM, de Vos CC, van Putten MJAM. Masking the auditory evoked potential in TMS-EEG: a comparison of various methods. Brain Topogr 2015;28:520-528.

52. Delorme A, Makeig S. EEGLAB: an open source toolbox for analysis of single-trial EEG dynamics including independent component analysis. J Neurosci Methods 2004;134:9-21.

53. Rogasch NC, Sullivan C, Thomson RH, et al. Analysing concurrent transcranial magnetic stimulation and electroencephalographic data: a review and introduction to the open-source TESA software. Neuroimage 2017;147:934-951.

54. Rogasch NC, Thomson RH, Farzan F, et al. Removing artefacts from TMS-EEG recordings using independent component analysis: importance for assessing prefrontal and motor cortex network properties. Neuroimage 2014;101:425-439.

55. Kayser J, Tenke CE. Principal components analysis of Laplacian waveforms as a generic method for identifying ERP generator patterns: I. Evaluation with auditory oddball tasks. Clin Neurophysiol 2006;117:348-368.

56. Oostenveld R, Fries P, Maris E, Schoffelen J-M. FieldTrip: open source software for advanced analysis of MEG, EEG, and invasive electrophysiological data. Comput Intell Neurosci 2011;2011:1-9.

57. Miocinovic S, de Hemptinne C, Chen W, et al. Cortical potentials evoked by subthalamic stimulation demonstrate a short latency HYPERDIRECT pathway in humans. J Neurosci 2018;38: 9129-9141.

58. Strafella AP, Vanderwerf Y, Sadikot AF. Transcranial magnetic stimulation of the human motor cortex influences the neuronal activity of subthalamic nucleus. Eur J Neurosci 2004;20: 2245-2249.

59. Benjamini Y, Yekutieli D. The control of the false discovery rate in multiple testing under dependency. Ann Stat 2001;29:1165-1188.

60. Benjamini Y, Hochberg Y. Controlling the false discovery rate: a practical and powerful approach to multiple testing. J R Stat Soc B Methodol 1995;57:289-300. 
61. Chen R, Classen J, Gerloff C, et al. Depression of motor cortex excitability by low-frequency transcranial magnetic stimulation. Neurology 1997;48:1398-1403.

62. Fecchio M, Pigorini A, Comanducci A, et al. The spectral features of EEG responses to transcranial magnetic stimulation of the primary motor cortex depend on the amplitude of the motor evoked potentials. PLoS One 2017;12:e0184910

63. Petrichella S, Johnson N, He B. The influence of corticospinal activity on TMS-evoked activity and connectivity in healthy subjects: a TMS-EEG study. PLoS One 2017;12:e0174879

64. Aeed F, Cermak N, Schiller J, Schiller Y. Intrinsic disruption of the M1 cortical network in a mouse model of Parkinson's disease. Mov Disord 2021;36:1565-1577.

65. Monje MHG, Blesa J, García-Cabezas MÁ, Obeso JA, Cavada C. Changes in thalamic dopamine innervation in a progressive Parkinson's disease model in monkeys. Mov Disord 2020;35:419-430.

66. Herz DM, Meder D, Camilleri JA, Eickhoff SB, Siebner HR. Brain motor network changes in Parkinson's disease: evidence from metaanalytic modeling. Mov Disord 2021;36:1080-1090.

67. Casula EP, Stampanoni BM, Pellicciari MC, et al. Subthalamic stimulation and levodopa modulate cortical reactivity in Parkinson's patients. Parkinsonism Relat Disord 2017;34:31-37.

68. Lehéricy S, Ducros M, Krainik A, et al. 3-D diffusion tensor axonal tracking shows distinct SMA and pre-SMA projections to the human striatum. Cereb Cortex 2004;14:1302-1309.
69. Blesa J, Trigo-Damas I, Dileone M, Del Rey NL-G, Hernandez LF, Obeso JA. Compensatory mechanisms in Parkinson's disease: circuits adaptations and role in disease modification. Exp Neurol 2017;298:148-161.

70. Julkunen P, Säisänen L, Hukkanen T, Danner N, Könönen M. Does second-scale intertrial interval affect motor evoked potentials induced by single-pulse transcranial magnetic stimulation? Brain Stimul 2012;5:526-532.

71. Sarasso S, D’Ambrosio S, Fecchio M, et al. Local sleep-like cortical reactivity in the awake brain after focal injury. Brain 2020;143: 3672-3684.

72. Ruan J, Bludau S, Palomero-Gallagher N, et al. Cytoarchitecture, probability maps, and functions of the human supplementary and pre-supplementary motor areas. Brain Struct Funct 2018;223: 4169-4186.

\section{Supporting Data}

Additional Supporting Information may be found in the online version of this article at the publisher's web-site. 


\section{SGML and CITI Use Only DO NOT PRINT}

\section{Author Roles}

(1) Research project: A. Conception, B. Organization, C. Execution; (2) Statistical analysis: A. Design, B. Execution, C. Review and critique; (3) Manuscript: A. Writing of the first draft, B. Review and critique.

G.L.: 1A, 1B, 1C, 2B, 3A

M.I.B.: $1 \mathrm{~B}, 1 \mathrm{C}, 3 \mathrm{~A}$

A.G.: $1 \mathrm{~A}, 1 \mathrm{C}, 1 \mathrm{~B}$

A.F.: $1 \mathrm{~B}, 2 \mathrm{~B}, 3 \mathrm{~B}$

L.R.: $1 \mathrm{~A}, 1 \mathrm{~B}, 3 \mathrm{~B}$

A.L.: 1B, 1C, 3B

G.P.: $1 \mathrm{C}, 3 \mathrm{~B}$

D.P.: $1 \mathrm{~B}, 3 \mathrm{~B}$

A.C.: $1 \mathrm{~A}, 3 \mathrm{~B}$

K.P.B.: $1 \mathrm{~A}, 3 \mathrm{~B}$

J.C.R.: $1 \mathrm{~A}, 3 \mathrm{~B}$

A.B.: $1 \mathrm{~A}, 1 \mathrm{~B}, 1 \mathrm{C}, 3 \mathrm{~B}$

\section{Full financial disclosures for the previous 12 months}

G.L.: none.

M.I.B.: none.

A.G.: none.

A.F.: none.

L.R.: none.

A.L.: none.

G.P.: none.

D.P.: none.

A.C.: none.

K.P.B. has received grant support from Horizon 2020 EU grant 634821 and honoraria/financial support to speak at/attend meetings from GSK, Boehringer-Ingelheim, Ipsen, Merz, Sun Pharma, Allergan, Teva, Lundbeck, and Orion Pharmaceutical. K.P.B. receives royalties from the Oxford University Press and a stipend for MDCP editorship. J.C.R.: none.

A.B.: none. 